\title{
4D surface kinematics monitoring through terrestrial radar interferometry and image cross-correlation coupling
}

\author{
Niccolò Dematteis ${ }^{\mathrm{a}, \mathrm{b}}$, Daniele Giordan ${ }^{\mathrm{a}, *}$, Francesco Zucca ${ }^{\mathrm{b}}$, Guido Luzi ${ }^{\mathrm{c}}$, Paolo Allasia ${ }^{\mathrm{a}}$ \\ a Geohazard Monitoring Group, Institute of Research for Hydro-Geological Protection, National Council of Research, Torino, Italy \\ ${ }^{\mathrm{b}}$ Department of Earth and Environmental Sciences, Università di Pavia, Pavia, Italy \\ ${ }^{\mathrm{c}}$ Geomatics Division, Centre Técnologic de Telecommunicacions de Catalunya, Castelldefels, Spain
}

\section{A R T I C L E I N F O}

\section{Keywords:}

3D motion

Surface kinematics

GBSAR

Image cross-correlation

Sensor network

Data integration

Interferometry

Glacier kinematics

Remote sensing

Monitoring

\begin{abstract}
A B S T R A C T
Complex gravitational phenomena can require terrestrial remote sensing solutions for monitoring their possible evolution, especially when in situ installations are not possible. This study merges terrestrial radar interferometry (TRI) and image cross-correlation (ICC) processing, which can detect complementary motion components, to obtain a 3-dimensional system able to measure the actual surface motion field of a pre-defined target. The coupling can be carried out on data acquired from different installations of the devices, and by applying specific transformations of the related coordinate systems. The data georeferencing is a critical issue that affects the correct spatial correspondence of the data and a new approach for georeferencing radar data is proposed. The result is a spatio-temporal $(3+1$-dimensional) high-resolution representation of the surface kinematics. The presented method has been tested for the measurement of the Planpicieux glacier surface kinematics (NW of Italy). The error analysis revealed a millimeter accuracy and precision of the measurement and a georeferencing uncertainty of a few metres.
\end{abstract}

\section{Introduction}

The necessity of strategies of preventive alert and risk mitigation related to gravitational slope processes, especially in mountain areas (e.g., landslides, avalanches, glacier outbursts, rock falls), requires systematic observation in order to a full understanding of these phenomena (Kääb et al., 2005). A particular attention is addressed to the surface kinematics monitoring, because in some cases it is possible to detect failure precursors (Faillettaz et al., 2015; Fukuzono, 1985).

Often, these processes are located in remote and harsh environments, consequently the access in these areas is usually difficult and perilous or even impossible. Therefore, various technologies and techniques have been developed and proposed for remotely monitoring gravitational slope phenomena (Arenson et al., 2016; Delacourt et al., 2007; Kääb et al., 2005; Leprince et al., 2008). Actually, the majority of the monitoring systems suffer some limitations. E.g., terrestrial radar and monoscopic photogrammetry can measure only partial components of the actual motion. Total stations and GPSs require the in-situ installation of artificial targets or sensors and they provide only punctual data. Other approaches produced digital elevation models (DEMs) and derived the kinematics through volume difference (e.g., structure-frommotion, SFM, and terrestrial laser scanner, TLS). Finally, space- or air- based remote sensing systems measure spatially distributed data over wide areas with high precision, though they require high financial costs and the survey repeatability is strongly limited; moreover, the measurement can be affected by unfavorable geometries.

In literature, different studies focused on remote sensing applications that can estimate the 3D surface deformations. Among the category of space-based systems, Wright et al. (2004) proposed a sort of multi-viewed stereoscopy of interferometric synthetic aperture radar (SAR) data that relies on the availability of SAR images acquired with four different look directions.

Concerning the ground-based systems, some studies proposed solutions for estimating the surface motion through the difference of DEMs acquired either by TLS (Bitelli et al., 2004) or by SFM (Piermattei et al., 2016; Roncella et al., 2014). But, cause the DEMs are defined on a 2D surface, $f(x, y)=z$, their difference provides 1D deformation measurements along the vertical axis (Monserrat and Crosetto, 2008). Monserrat and Crosetto (2008) proposed an algorithm of surface matching able to provide 3D deformations that proved to perform well for TLS data acquired from short distances. However, the main drawback of the TLS acquisitions remains the limited revisiting time of the measurement. Manconi et al. (2013) proposed an estimate of the 3D deformation by spatially interpolating over a DEM the punctual data

\footnotetext{
* Corresponding author.

E-mail address: daniele.giordan@irpi.cnr.it (D. Giordan).
} 
acquired by a total station. Other works projected the data acquired by GBSAR (Dematteis et al., 2017; Luzi et al., 2007), satellite SAR (Huang and $\mathrm{Li}, 2011$ ) or produced through image cross-correlation (ICC) (Gabrieli et al., 2016; Giordan et al., 2016) along the expected maximum gradient direction or the expected flow direction (Schwalbe and Maas, 2017). Similarly, Ahn and Box (2010), Messerli and Grinsted (2015), and Travelletti et al. (2012) applied the image orthorectification and consequently adjusted the ground sampling distance (GSD) of each pixel according to the incident angle. Both approaches made the strong assumption of the invariance of the surface morphology during the survey (Travelletti et al., 2012), though this might be not valid in presence of quite active phenomena or measurements separated by long time intervals. Schwalbe and Maas (2017) adopted a multi-camera network to produce updated DEM integrated in the processing chain.

In recent years, the research is focusing on the integration of different types of monitoring systems; thereby it is possible to overtake the limitations of the single devices and acquire information that are more representative of the phenomenon. Kenner et al. (2014) merged airborne LiDAR data to assess vertical deformations by means of DEM difference with aerial photogrammetry for detecting horizontal movements through ICC. Also, TLS and terrestrial radar interferometry (TRI) were coupled for monitoring 3D deformations of archaeological monuments (Tapete et al., 2013), buildings (Marambio et al., 2009) and cliff evolution (Lim et al., 2005). Piermattei et al. (2016) used groundbased photogrammetry and Scaioni et al. (2017) combined unmanned aerial vehicles (UAVs) and ground-based photogrammetry for assessing the evolution of 3D glacial processes, in particular related to mass balance change. Malet et al. (2016) monitored a set of landslides adopting a network of permanent GPSs, TLS, and photogrammetric cameras.

In the present paper, we propose a method for measuring the actual $3+1$ D surface kinematics of a gravitational slope phenomena through the coupling of the data collected by two different ground-based remote sensing systems, (i) a ground-based SAR (GBSAR) and (ii) a monoscopic visual-based system (VBS). Their data are processed respectively through radar interferometry and image cross-correlation (ICC) techniques. The 3-dimensional reconstruction of the motion vector is possible because the devices observe different and complementary motion components: the line of sight (LOS) -parallel component is measured by the GBSAR, while the VBS detects the two components orthogonal to the LOS. One of the objectives of our approach is the application of the method to purely remote sensing measurements from medium range (i.e., a few thousand metres).

Furthermore, we present the method application and results related to a 24 days-long campaign surveying the Planpincieux glacier that was carried out on September 2015. The conclusive part of the paper is dedicated to some considerations and comments concerning the proposed method.

\section{Dataset and study site}

The data presented in this paper were collected during the period 04 - 27 September 2015. The goal of the study was the measurement of the surface displacements of the Planpincieux glacier that is placed on the southern side of the Mont Blanc massif, in the Italian Alps (Fig. 1).

The Planpincieux glacier is a polythermal glacier with elevation ranging between 2500 and $3500 \mathrm{~m}$. This paper deals with the analysis of the data related to the lower part of the glacier, which is classified as a "temperate terrace avalanching glacier" (Faillettaz et al., 2015; Giordan et al., 2016; Pralong, 2005). It reaches an altitude of approximately $2900 \mathrm{~m}$ and it is composed of two main icefalls separated by a central ridge of partly-exposed bedrock. The western side is the most active and shows a highly crevassed texture with a mean slope of $32^{\circ}$; it is characterized by a frontal $20 \mathrm{~m}$-high vertical ice wall just above a steep bedrock face from which several failures of ice are triggered periodically during the warm season. In the past, several major ice avalanches and outbursts of water pocket occurred, and in some cases they threatened the underlying village of Planpincieux and damaged the road.

The entire body of the Planpincieux glacier is continuously monitored for research and civil protection purposes since 2013 by a monoscopic visual-based system (VBS) that is installed on the opposite valley side w.r.t. the glacier, at an elevation of $2305 \mathrm{~m}$ and a distance of approximately $3800 \mathrm{~m}$ (Giordan et al., 2016). It consists of two consumer-grade cameras with APS-C sensors of $18 \mathrm{Mpx}$ that are equipped with $297 \mathrm{~mm}$ (TELE module) and $120 \mathrm{~mm}$ (WIDE module) focal lens (camera specifications are provided in Table 1).

The cameras are installed on a concrete plinth that is placed inside a plastic shelter box. An energetic module composed of two solar panels supplies electricity to the VBS, therefore the system can work autonomously and it is completely controlled by remote; both modules of the VBS acquire one image per hour. Since shadows and illumination changes can deeply affect the results of the ICC (Ahn and Box, 2010; Debella-Gilo and Kääb, 2011; Giordan et al., 2016; Messerli and Grinsted, 2015; Piermattei et al., 2016; Travelletti et al., 2012), we manually selected for the processing one image per day acquired between 5 and $7 \mathrm{pm}$. During the period of the study, adverse weather conditions prevented the correct acquisition of some data, 21 of 25 and 20 of 25 images of the TELE and WIDE modules respectively were adopted for the processing.

During the same period, a commercial Ku-band (IBIS-L) groundbased SAR (GBSAR) was installed in the Planpincieux hamlet at the elevation of $1582 \mathrm{~m}$. The mean distance from the glacier was approximately $2500 \mathrm{~m}$. That positioning aimed at maximizing the parallelism between the LOS and the expected motion direction of the western icefall; also, a portion of the eastern tongue was visible from the GBSAR location. The radar acquired one image every $16 \mathrm{~min}$ and it collected more than 2200 images during the time span of the experimental setup.

Furthermore, a 1-m resolution digital elevation model (DEM) was available for the study, which was acquired by an airborne LiDAR survey in June 2014; during the LiDAR measurement, it was acquired also a high-resolution orthophoto of the glacier.

For further details concerning the site study and the datasets refer to Dematteis et al. (2017) and Giordan et al. (2016).

\section{Methods}

The method we propose enables estimating the glacier surface deformations measured by GBSAR and VBS devices. These instruments can detect different and complementary components of the motion vector; therefore, their results can be coupled to reconstruct the actual 3D surface kinematics.

Overall, the data processing is composed by three main procedures: (i) interferometric processing of the radar images; (ii) image crosscorrelation (ICC) of the photographic data and (iii) georeferencing and coupling of the results of the two different instruments. The workflow of the method is depicted in Fig. 2.

\subsection{Image cross-correlation}

Digital image processing methodologies for measuring fluid motion fields were initially developed in the ' 90 s for particle images velocimetry (PIV) (Willert and Gharib, 1991). Applications in earth sciences are more recent and they aim at estimating the deformations of natural active surfaces such as landslides or glaciers with the support of airborne (Debella-Gilo and Kääb, 2011; Kääb et al., 1998; Scherler et al., 2008) and ground-based images (Ahn and Box, 2010; Fallourd et al., 2010; Giordan et al., 2016; Messerli and Grinsted, 2015; Schwalbe and Maas, 2017; Travelletti et al., 2012; White et al., 2003). Similar approaches were also applied to non-visual images, e.g., radar amplitude maps (Casu et al., 2011; Strozzi et al., 2002) or interferometric maps (Raucoules et al., 2013). 


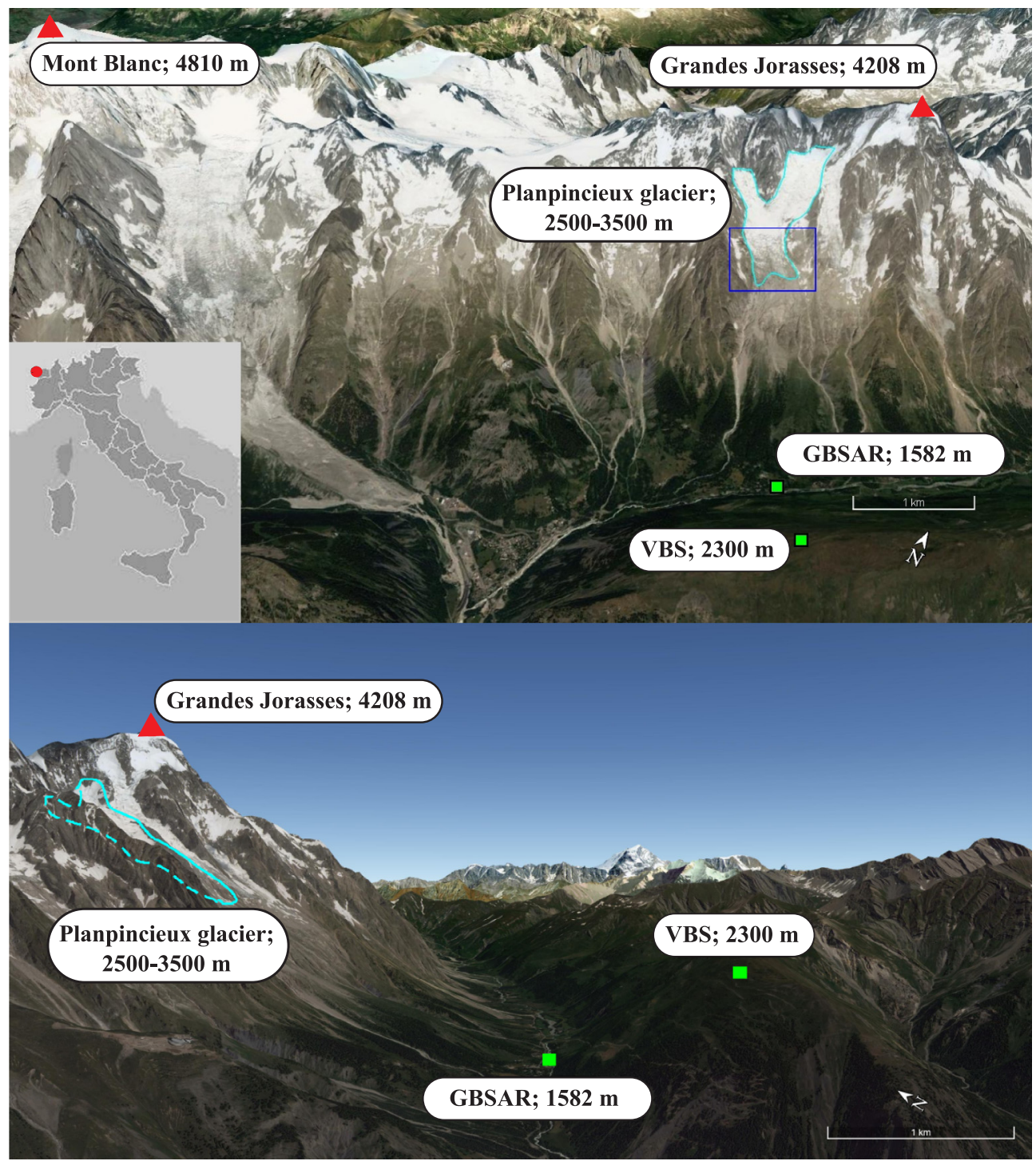

Fig. 1. Overview of the study area. The entire Planpincieux glacier is highlighted in sky blue and the lower part is surrounded by a blue box. The locations of the GBSAR and visual-based station are depicted as green squares.

Table 1

DSLR camera specifications.

\begin{tabular}{|c|c|c|c|c|c|c|}
\hline & $\begin{array}{l}\text { Camera } \\
\text { model }\end{array}$ & Sensor & Resolution (px) & $\begin{array}{l}\text { Focal } \\
\text { length } \\
(\mathrm{mm})\end{array}$ & ISO & Aperture lens \\
\hline TELE & $\begin{array}{l}\text { Canon } \\
\text { EOS 600D }\end{array}$ & $\begin{array}{l}\text { CMOS } \\
\text { APS-C }\end{array}$ & $3456 \times 5184$ & 120 & 200 & $\mathrm{f} / 8$ \\
\hline WIDE & $\begin{array}{l}\text { Canon } \\
\text { EOS 100D }\end{array}$ & $\begin{array}{l}\text { CMOS } \\
\text { APS-C }\end{array}$ & $5184 \times 3456$ & 297 & 200 & $\mathrm{f} / 8$ \\
\hline
\end{tabular}

Usually, the surface motion is evaluated by computing the crosscorrelation between a template image and a searching image; the displacement is then estimated according to the location of the maximum correlation coefficient.

In this study, we computed the ICC in the frequency domain (often referred as phase correlation) via discrete Fourier transform (DFT) with the algorithm proposed by Guizar-Sicairos et al. (2008), which allows a cost-efficient sub-pixel offset estimation with a two-step matrix-multiply; that operation strongly limits the amount of zero-padding, thus reducing the computational costs. That matching algorithm runs two passes of the DFT, after the first pass it shifts the center of the interrogation area according to the obtained pixel offset and then runs the DFT only in the neighourhood of a limited subset of the data to achieve a finer accuracy. This solution reduces the loss of information caused by the displacement of the searching area that usually affects phase correlation algorithms (Thielicke and Stamhuis, 2014). Other methods, such as the minimum quadratic difference (Gui and Merzkirch, 1996), as well as the direct cross-correlation (Keane and Adrian, 1992) were considered but not applied because they have higher computational costs. Pust (2000) and Thielicke and Stamhuis (2014) estimated a difference of the computational time of two orders of magnitude for searching windows of 128 px between phase crosscorrelation and direct cross-correlation.

The image processing chain adopted in our work consists in five main procedures: (i) manual images selection, (ii) illuminant correction, (iii) co-registration, (iv) pixel offset matching and (v) automate outlier detection. Fig. 3 we illustrates the steps of the ICC chain.

The first step concerned the manual selection of one image per day to obtain a sequence with homogeneous and uniform illumination and visibility conditions (Ahn and Box, 2010; Debella-Gilo and Kääb, 2011; Giordan et al., 2016; Messerli and Grinsted, 2015; Piermattei et al., 2016). Therefore, we selected the images acquired when the sun did not lit the glacier surface directly and the diffuse illumination prevailed; in 


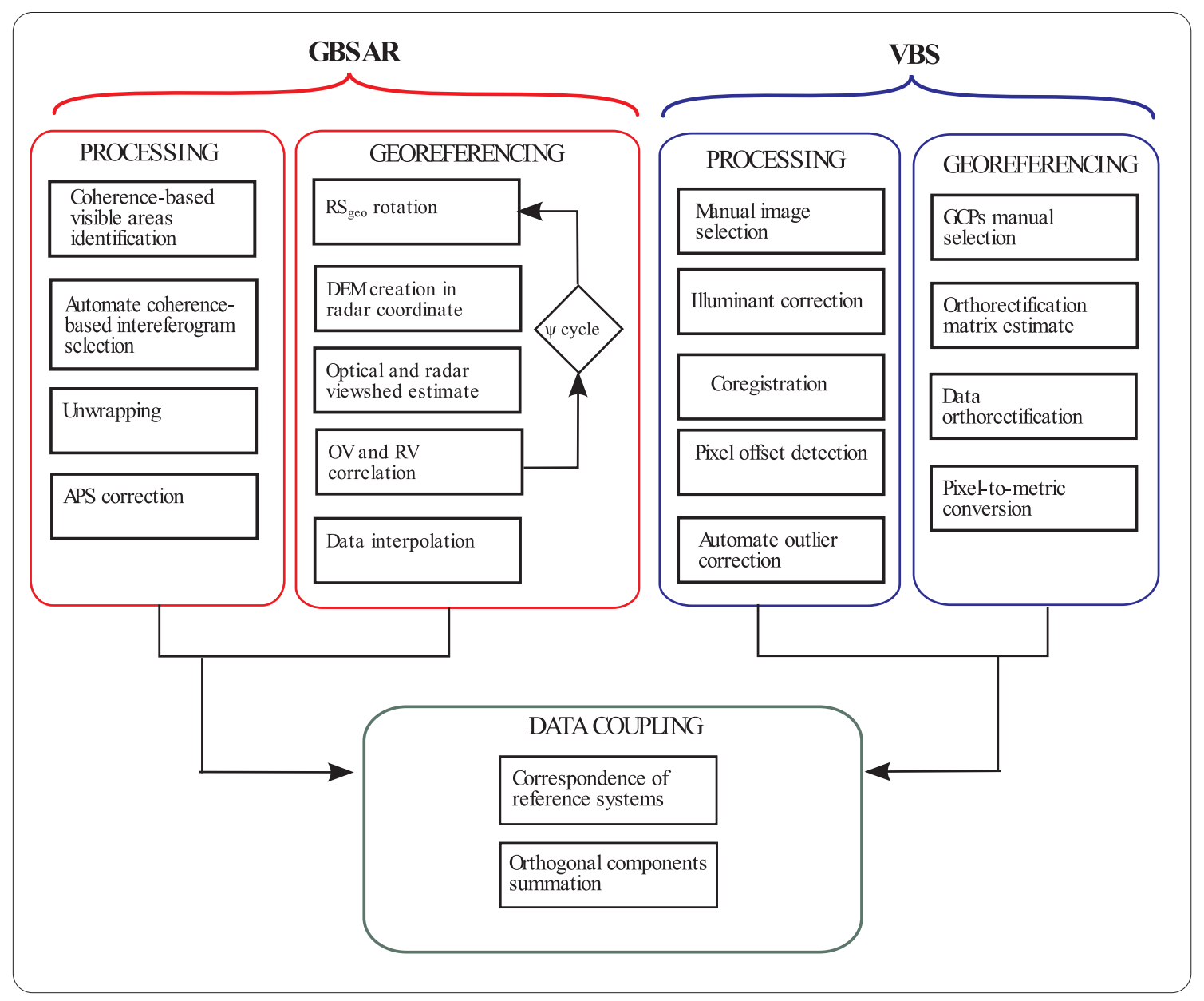

Fig. 2. Workflow of the proposed method for 3D data coupling.

particular, we used pictures taken during the late-afternoon hours (i.e., 5-7 pm). Thus, the shadow changes due to the surface roughness had a minimum impact on the cross-correlation computation.

Next, we estimated the scene illuminant with principal component analysis (Cheng et al., 2014) and then we subtracted it from the scene; thereby, we further make the illumination conditions more uniform among the different images.

In the third step, all the images were coregistered with respect to (w.r.t.) a common image (i.e., the first image of the stack) in order to correct possible movements of the camera or optical path changes. The matching algorithm was applied to an area of stable surface (i.e., bedrock) and the images were translated in the vertical and horizontal directions according to the obtained pixel offsets; most coregistration absolute values ranged between 0 and $5 \mathrm{px}$, only in a few images the horizontal offset was approximately $10 \mathrm{px}$. Further analysis did not reveal image rotations.

The subsequent operation was the displacement assessment by means of the sequential ICC computation on chips identified by a sliding window. For the TELE images, we adopted a 256 px-side sliding window with $75 \%$ overlapping (i.e., sliding steps of $64 \mathrm{px}$ ), while for the WIDE images the window size and sliding step were of $128 \mathrm{px}$ and $64 \mathrm{px}$. Thereby obtaining a motion field mapped on a $64 \mathrm{px}$-side grid for both cases.

Finally, we dealt with the refinement of the results. Multiple sources of error can intervene during the processing (i.e., shades and shadows, non-optimal illumination, image defocusing or morphological surface
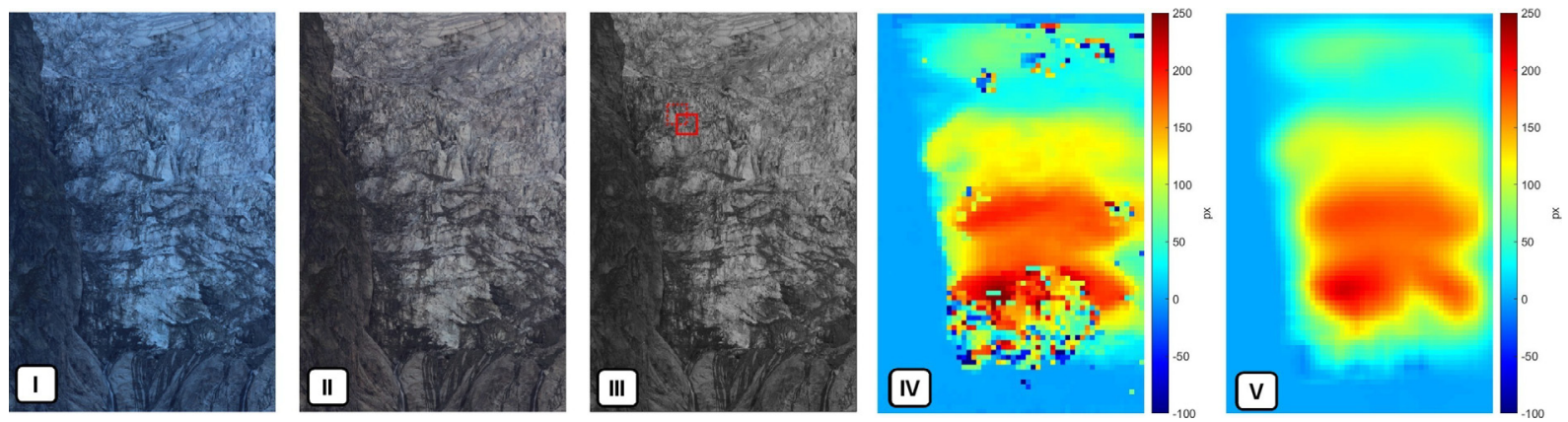

Fig. 3. Step sequence of the image processing method: (i) the raw image is acquired, (ii) the illuminant is removed, (iii) the image is converted in grey scale and coregistered, then (iv) the ICC is computed with the sliding window obtaining the raw results, finally (v) the automate outlier detection and removal is operated. The picture was acquired by the TELE module on 06/09/2015 at h19:00. 

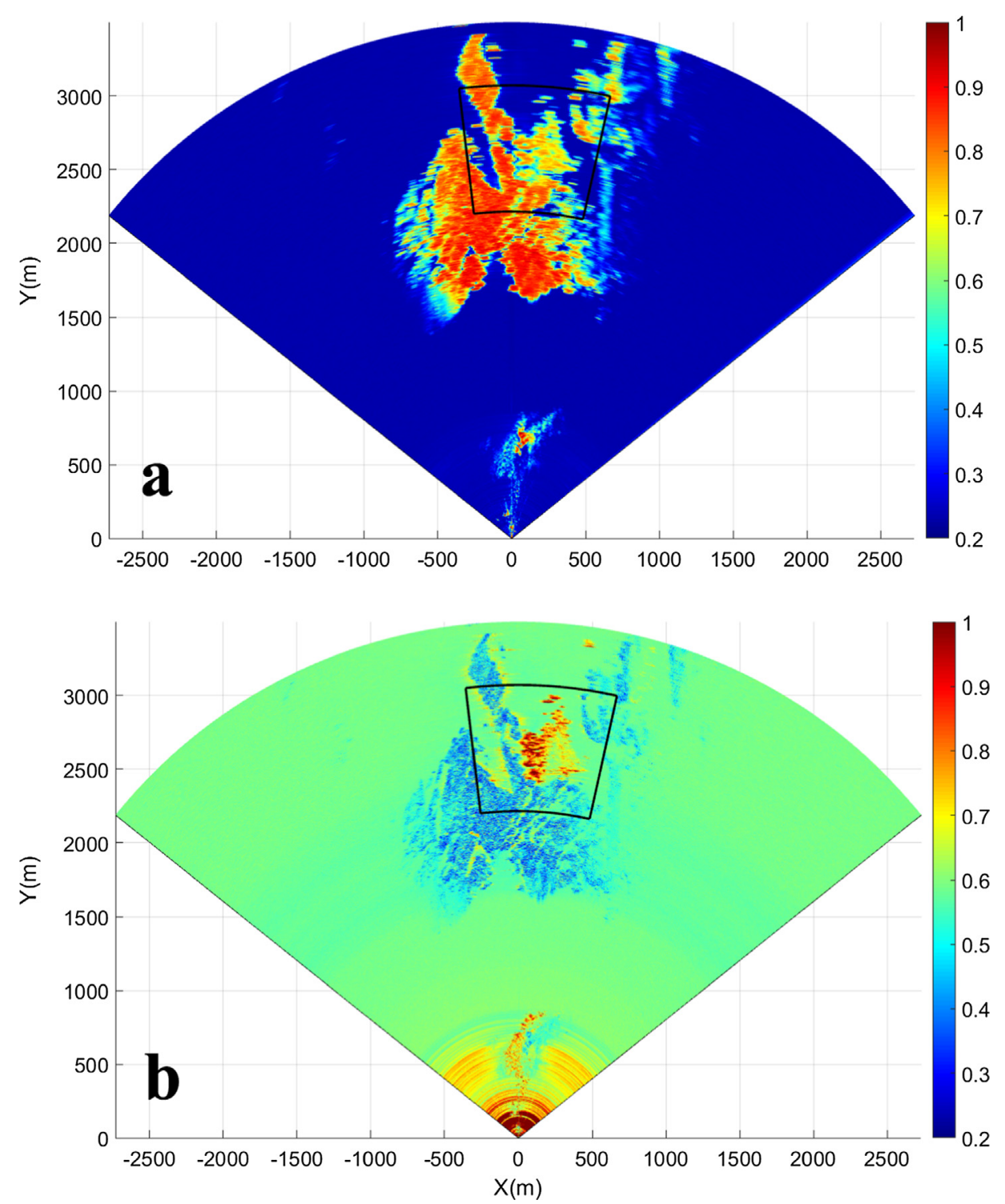

Fig. 4. (a) Map of mean temporal coherence $(\bar{\gamma})$ and (b) map of amplitude dispersion (DA) of the backscattered signal of the GBSAR. The investigated area is included in the black box; the glacier area is characterized by high values of DA, while bedrock displays lower values. Low values of $\bar{\gamma}$ are associated to areas in shade.

changes) often difficult to detect and evaluate separately (Gabrieli et al., 2016). Actually, correcting the correlation anomalies is a crucial step to obtain reliable results. Various methods have been proposed to this aim, some studies adopted optimized methods to remove the outliers directly during the correlation computation (Ahn and Box, 2010; Hart, 1998), while other approaches rely on empirical or statistical analysis in the post-interrogation phase (Debella-Gilo and Kääb, 2011; Giordan et al., 2016; Westerweel and Scarano, 2005). We opted for the normalized median test proposed by Westerweel and Scarano (2005); it consists in the analysis of the parameter $r_{0}$ defined through the following equation

$r_{0}=\frac{\left|U_{0}-U_{m}\right|}{r_{m}+\epsilon}$

where $U_{0}$ is the investigated datum, $U_{m}$ is the median of the $5 \times 5$ neighbours of $U_{0},\left\{U_{1}, U_{2}, \cdots, U_{24}\right\}$, excluding $U_{0}$ from the computation, $r_{m}$ is the median of the residuals defined as $r_{i}=\left|U_{i}-U_{m}\right|$, with $U_{i}=\left\{U_{1}, U_{2}, \cdots, U_{24}\right\}$, and $\epsilon$ is an offset introduced in case $r_{m}$ was too close to zero. Westerweel and Scarano (2005) found out that a value of $r_{0}>2$ corresponds approximately to the $90^{\text {th }}$-percentile of the residuals, whatever the data values or distributions are and it is rather insensitive to the window size. That means that the method can be considered a "universal outliers' detection". They also suggest the value of 0.1 for the $\epsilon$ term.

After outliers rejection, missing data were estimated by Natural Neighbour interpolation (Sibson, 1981) and a low-pass filter was applied to the resulting maps for taking into account the sliding window overlapping; the averaging filter had $7 \times 7$ and $3 \times 3$ side for the TELE and WIDE images respectively.

\subsection{Interferometry}

TRI proved to be a valuable tool in the monitoring of glaciers (Allstadt et al., 2015; Noferini et al., 2009; Riesen et al., 2011). In our study, we performed the interferometric processing to detect the surface motion of the glacier along the LOS direction.

Radar interferometry is the process that analyses the differential phase between two different images, aiming at estimating the displacement occurred during the two acquisitions. For the sake of completeness, the basic theory of interferometry is briefly described in the following. For a more exhaustive explanation of the SAR interferometry we refer to Bamler and Hartl (1998) and Rosen et al. (2000); while in 
Caduff et al. (2015); and Monserrat et al. (2014) it is possible to find recent reviews of the TRI literature.

Let consider the electromagnetic wave scattered by a single target; the distance covered by the emitted signal is $R=\mathrm{n} \lambda+\frac{\lambda}{2 \pi} \phi$, where $\lambda$ is the wave length and $\phi$ is the phase. Therefore, a motion occurred between two acquisitions is equal to

$R_{2}-R_{1}=\frac{\lambda}{4 \pi}\left(\phi_{2}-\phi_{1}+2 \mathrm{n} \pi\right)=\frac{\lambda}{4 \pi}(\Phi+2 \mathrm{n} \pi)$

where $2 n \pi$ belongs to the intrinsic ambiguity of the phase periodicity (i.e., phase wrapping).

Actually, in the cases when the radar is maintained in the same position (zero baseline), the interferometric phase is given by the sum of different terms, that is

$\Phi=\varphi_{d}+\varphi_{a}+\varphi_{s}$

where $\varphi_{d}$ is the phase directly related to the displacement of the target, $\varphi_{a}$ is due to the atmospheric condition change (often named atmospheric phase screen, APS), which alters the optical path of the electromagnetic wave, and $\varphi_{s}$ is the term including thermal noise and phase change caused by the variation of the scattering properties (Luzi et al., 2004). Accordingly, some data processing is necessary to determine the contribute of the different terms and correctly estimate $\varphi_{d}$.

In our work, we discriminated the areas illuminated by the radar signal from the shadowed regions. To this aim, points displaying a mean temporal coherence $\bar{\gamma}$ lower than an empirical threshold (i.e., $\bar{\gamma}<0.55$ ) were masked out (Fig. 4). In other studies, the selection of the scattering points was driven by an amplitude dispersion- (DA) based criterion (Ferretti et al., 2001). Ice metamorphisms and snow deposition introduced strong changes of the dielectric properties of the target, causing high variability of the scattered signal amplitude and yielding substantial values of DA. In fact, the DA observed on the glacier area displayed values ranging between 0.4 to more than 1 (Fig. 4). Therefore, a DA-based criterion to select shaded areas was not suitable.

The second operation aimed at resolving the phase ambiguity by applying a 2-D unwrapping algorithm (Costantini, 1998). Such algorithm introduced different phase offsets among not connected regions. Therefore, it was necessary to manually identify the main compact areas and then subtract the corresponding phase offsets. That operation further reduced the unwrapping errors and significantly improved the result accuracy and precision.

Subsequently, the interferograms affected by strong decorrelation were discarded by the temporal stack of the interferograms, thereby reducing errors due to noise or residual phase wrapping. The huge amount of data made necessary to establish an automate criterion to reject decorrelated interferograms; therefore, we established an empirical threshold of the mean spatial coherence $\langle\gamma\rangle<0.65$ to identify the interferograms to be discarded (Dematteis et al., 2017).

Eventually, we dealt with the APS removal. The glacier was located $1200 \mathrm{~m}$ higher than the GBSAR positioning and it was characterized by significant elevation changes in the investigated area. As a consequence, the atmospheric variables, which drive the optical path length of the electromagnetic wave, might significantly vary and thus affect the measurement of the interferometric phase. Hence, being that for stable areas we expected $\varphi_{d}=0$ and $\mathbb{E}\left\{\varphi_{s}\right\}=0$ (i.e., white noise), the value of $\varphi_{a}$ was estimated with a polynomial regression of degree two of the interferometric phase observed on 5190 points, corresponding to the stable areas (i.e., points with $D A<0.35$ ); the elevation coordinates were introduced as an explicit variable to take into account the topographic influence on the atmosphere (Dematteis et al., 2017; Iglesias et al., 2014; Noferini et al., 2005).

The results of the interferometric process are represented on the radar maps with a constant range resolution (i.e., $0.43 \mathrm{~m}$ ). Conversely, the azimuth resolution varies linearly with the range and it is approximately $11 \mathrm{~m} @ 2500 \mathrm{~m}$. As a result, the radar data are mapped on a grid with non-uniform resolution.

\subsection{Data georeferencing and coupling}

TRI and ICC provide different and complementary motion components that can be merged to obtain a 3-dimensional representation of the surface motion. The necessary conditions to couple the different data is that they must be represented in the same coordinate system (CS). Therefore, (i) the reference axes must be parallel and (ii) the map resolutions of the data must be equal.

The former condition is automatically achieved if the two measurement systems observe the target area with the same LOS. Conversely, when the systems acquire from different spots, their CSs must be geometrically transformed in order to make their axes parallel. In addition, in this second case, the motions measured by the different systems are not orthogonal, thus it is necessary to identify only the linearly independent components to obtain the actual 3D motion vector. The second necessary condition can be achieved through the application of spatial interpolation on a regular grid. Therefore, it is required the support of a DEM on which the data are projected; in our case, we used a 1-m resolution DEM. Finally, the georeferencing allows identifying the corresponding data with the same spatial coordinates.

Hereafter we will refer to $C S_{\text {geo }}$ as the 3D geographic CS in UTM $32 \mathrm{~T}$ (WGS84) coordinates $(X, Y, Z) ; C S_{v}$ stands for the 2D CS of the visual images, expressed in pixel coordinates $(u, v)$; and $C S_{r}$ for the 2D radar CS with local Cartesian coordinates $\left(x, y^{*}\right)$. Here and in the following the apex * is used to highlight that the related coordinates are not horizontal.

\subsubsection{Photographic images}

A transformation matrix was computed using an image orthorectification procedure to obtain a correct georeferencing of the ICC displacement map. The photographic images were handled through the orthorectification process that allowed projecting the photographs on a $3 \mathrm{D}$ surface (i.e., the DEM) adjusting the deformations due to topography and perspective, thereby the scale of the image was constant when it was projected in the new CS. The principle of the image orthorectification is based on the pinhole camera model, according to which, each point in the real 3D world is projected in the 2D image plane by a straight line passing through the ideal centre of the camera.

It is well known (Luhmann, 2009) that the necessary camera external and internal parameters to operate the image orthorectification can be estimated by the knowledge of the coordinates in the real 3D world and in the $C S_{v}$ of at least six corresponding points within the observed area. The most common approach consists in measuring the coordinates of a set of ground control points (GCPs) clearly recognizable within the photograph by means of GPSs or other topographic instruments; sometimes, this is achieved by placing artificial targets within the study area (Aguilar et al., 2008; Messerli and Grinsted, 2015; Schwalbe and Maas, 2017; Travelletti et al., 2012).

Instead, our work aimed at measuring surface deformations purely from remote, thus avoiding any access in the studied area, including the installation of artificial GCPs. Therefore, we manually selected a set of 38 and 35 recognizable features in the TELE and WIDE images respectively, such as bedrock cracks, pinnacles or sharp edges (Messerli and Grinsted, 2015; Piermattei et al., 2016). We had available TELE photographs taken on July 2014 (i.e., contemporary to the DEM and orthophoto acquisitions), therefore we could also identify features of the glacier morphology, such as crevasses and seracs, thus allowing a more uniform GCP distribution on the scene. For the WIDE images, we recognized features only on the stable bedrock surrounding the entire glacier (Fig. 5). The geographical coordinates were extracted from the orthophoto and the DEM acquired during the 2014 LiDAR survey.

We performed the image orthorectification using the open-source Matlab $^{\mathrm{TM}}$ toolbox, ImGRAFT (Messerli and Grinsted, 2015); the algorithm estimates the camera parameters in order to minimize the squared projection error of the GCPs.

Eventually, the transformation matrix was applied to the 


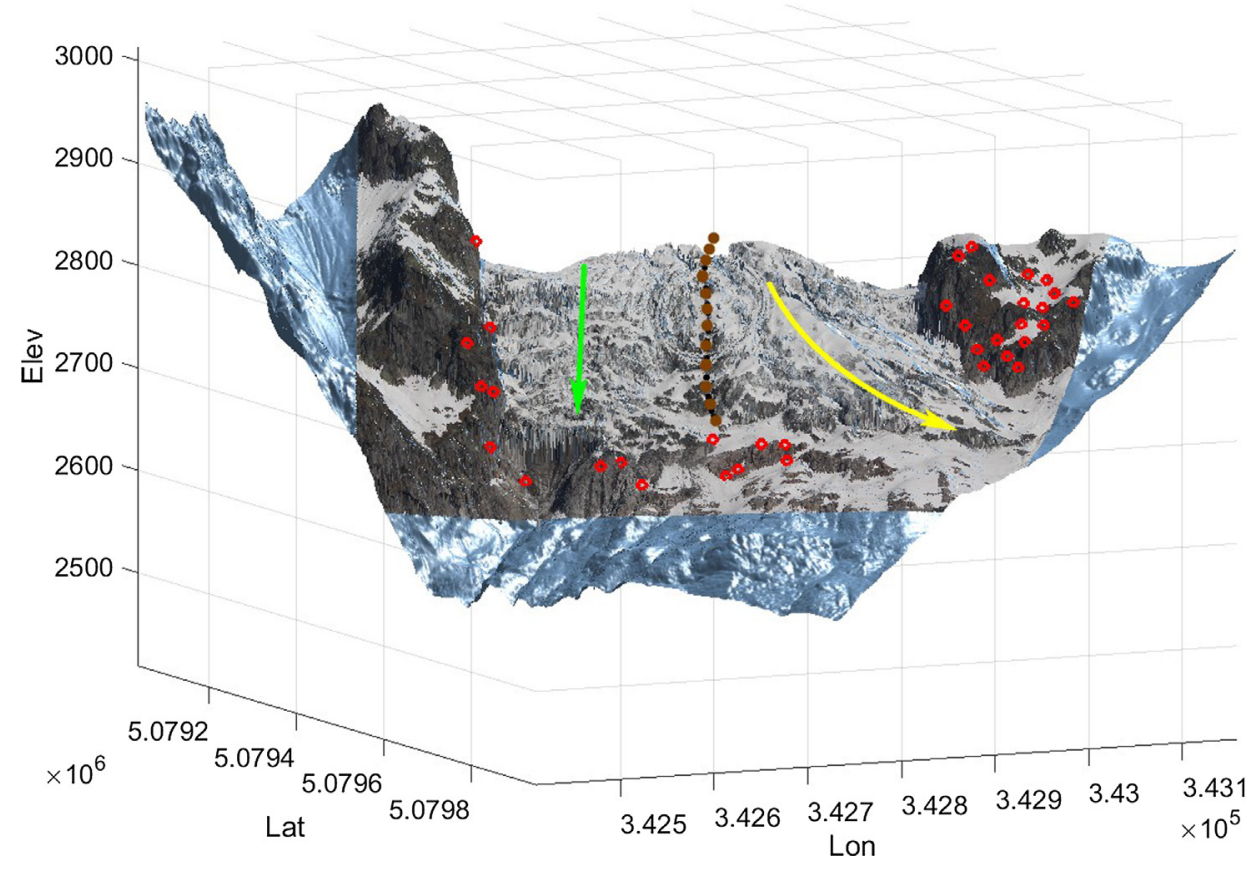

Fig. 5. WIDE image orthorectified and represented projected onto the DEM. GCPs are reported as red circles. The camera position is frontal to the western tongue (green arrow), thus the vertical motion component prevails. Conversely, the eastern tongue (yellow arrow) flows diagonally w.r.t. the LOS. A ridge of bedrock, highlighted by brown dots, separates the two tongues. displacement maps obtained during the ICC processing and then the motion expressed in pixel was converted to metric units. To attain that, we computed the ground sampling distance (GSD) projected on the plane orthogonal to the LOS.

$G S D=2 D \cdot \tan \left(\operatorname{atan} \frac{(S / 2 f)}{R}\right)$

where $D, S, R$ and $f$ are respectively the target distance, the sensor size, the images resolution (in px) and the focal length of the optical system.

According to Eq. (4), the GSD increased linearly with the distance and it varied across the images with values ranging between approximately $5.0-5.9 \mathrm{~cm}$ and $12.4-14.5 \mathrm{~cm}$ for the ZOOM and WIDE images respectively. Hence, considering that the tiles of the motion map had a side of 64-px, the mean tile sizes were roughly $3.5-\mathrm{m}$ side and $8.7-\mathrm{m}$ side respectively for the TELE and WIDE data. At each tile was associated a single value of surface motion.

We remark that the GSD computation in Eq. (4) corresponds to the pixel footprint on a plane orthogonal to the LOS and that the only variable is the distance $D$, while the other terms are fixed parameters. Therefore, the pixel-to-metric conversion (often called scaling) is rather insensitive to the possible morphological changes of the surface, because the distance change is likely to be negligible w.r.t. $D$; e.g., a hypothetical distance variation of $10 \mathrm{~m}$ would yield a relative error of $0.2 \%$. By contrast, the estimate of the adjusted GSD in accordance with the incident angle of the optical ray on the surface is subjected to significant errors when dealing with not updated DEMs or in situations that involve high morphology change (Gabrieli et al., 2016; Messerli and Grinsted, 2015; Travelletti et al., 2012).

\subsubsection{Interferometric data}

For what concerns the georeferencing of the radar data, a common procedure consists in identifying on the radar maps a set of artificial GCPs with known coordinates, such as passive (Marambio et al., 2009) or active (Lingua et al., 2008) corner reflectors (CRs), in order to estimate the unknown transformation parameters and then interpolate on the DEM with the least squared method. We propose a different solution that can be adopted in cases where neither the installation of artificial CRs nor the identification of high reflective areas with known coordinates are feasible, a situation quite occurring when monitoring inaccessible areas.
Let first describe the characteristics of the radar maps, whose Cartesian coordinates are $\left(x=r \cos \alpha, y^{*}=r \sin \alpha\right)$, where $\alpha$ is the azimuth angle and $r$ is the range. Actually, given the vertical aperture of the radar beam and the elevation angle of the LOS that can be non-null, $y^{*}$ is the curvilinear surface $y^{*}=\sqrt{y^{2}+z^{2}}$, where $z$ is the height of the target w.r.t. the radar position and $y$ is the horizontal projection of $y^{*}$. Therefore, $y$ and $z$ must be determined for correctly georeferencing the radar data in a $3 \mathrm{D}$ space.

The surface in geographic coordinates $f\left(X, Y^{*}\right)=Z$ is know, where $Y^{*}$ is the equivalent of $y^{*} ; f$ it is well defined as $X$ and $Y^{*}$ are linearly independent. Hence, it is possible to estimate $z$ with Natural Neighbour Interpolation (Sibson, 1981) by evaluating $f\left(x, y^{*}\right)=z$; the $y$ coordinate is estimated consequently with $y=\sqrt{r^{2}-x^{2}-z^{2}}$.

The method above can be applied only if the axes origins of $C S_{r}$ and $C S_{\text {geo }}$ coincide and the respective axis are parallel. Therefore, when this condition is not fulfilled, it is first necessary to rototranslate the geographical coordinates according to the angle $\psi$ included between the radar LOS and the geographic north.

In optimal contexts, $\psi$ can be estimated by measuring the coordinates of the radar edges by means of GPSs. We propose an alternative method that is suited in situations where accurate GPS measurements are not available and $\psi$ is only approximately assessed or even unknown; moreover, we will explain that the uncertainties are comparable to the GPS-based method, or even lower especially for long range measurements.

Let consider the optical viewshed (OV) (i.e., the visible geographical area) from the radar position that can easily computed from the DEM (Messerli and Grinsted, 2015), actually it corresponds with the area illuminated by the radar microwave bundle. The areas visible by the radar return a high amplitude backscattered signal (Tapete et al., 2013); therefore, the "radar viewshed" (RV) can be estimated as the areas on the radar maps with the mean amplitude greater than an empirical threshold (Fig. 6). The amplitude is expressed in $\mathrm{dB}$ w.r.t. the environmental noise.

Consequently, the OV and RV differ mainly for the map resolution where they are projected. The method we developed for estimating $\psi$ consists in varying the value of $\psi$ within a cycle whose each iterate is composed by three steps: (i) first, the DEM coordinates are rototranslated according with the current value of $\psi$ (being $\left(x_{r}, y_{r}, z_{r}\right)$ the GBSAR positioning in UTM coordinates) 

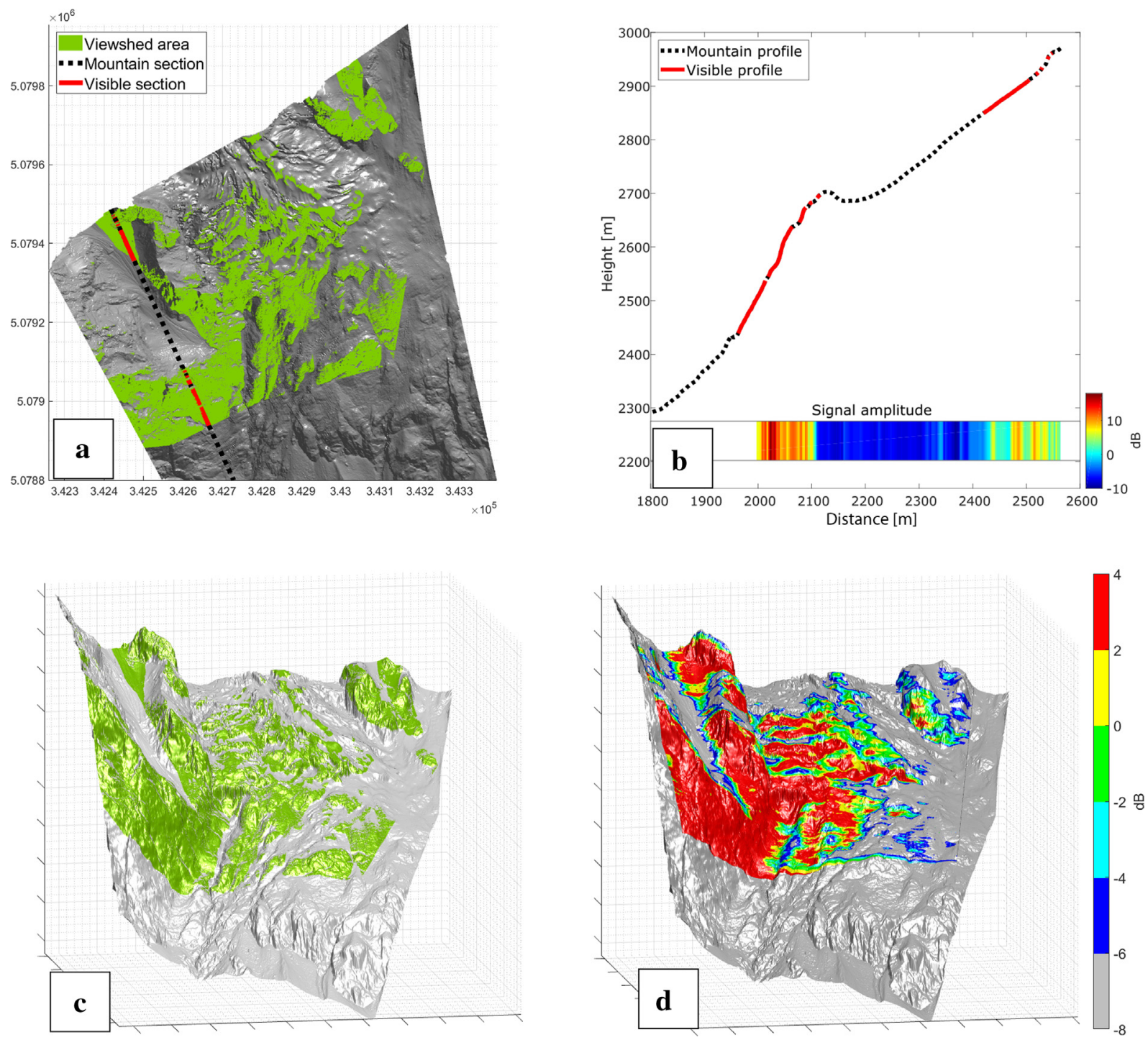

Fig. 6. (a) The optical viewshed is depicted in green on the DEM. The solid red line is the visible part of the slope section (black dashed line) from the GBSAR location. (b) The mountain profile of the selected section is represented (black dashed line) with the visible part in red (solid line). In the lower part is reported the signal amplitude corresponding to the DEM section. The high amplitude values in correspondence of the visible profile are evident. In the blank part, the amplitude was not processed. (c) Maps of optical viewshed and (d) mean amplitude map of the radar backscattered signal; the different empirical thresholds adopted to estimate the viewshed correlation are depicted.

$\left\{\begin{array}{c}X^{\prime}(\psi)=X \cos (\psi)+Y \sin (\psi)-x_{r} \\ Y^{\prime}(\psi)=X \sin (\psi)-Y \cos (\psi)-y_{r} \\ Z^{\prime}=Z-z_{r}\end{array}\right.$

(ii) Second, the RV map is interpolated (Natural Neighbour Interpolation) on the DEM in order to have the $\mathrm{RV}_{\text {interp }}$ and $\mathrm{OV}$ with the same uniform spatial resolution.

$g\left(x, y^{*}\right)=\mathrm{RV} \mapsto g\left(X^{\prime}(\psi), Y^{*^{\prime}}(\psi)\right)=\mathrm{RV}_{\text {interp }}(\psi)$

(iii) Third, we compute the spatial correlation between the $\mathrm{RV}_{\text {interp }}(\psi)$ and OV maps.

$\rho=\operatorname{corr}\left[\mathrm{OV}, \mathrm{RV}_{\text {interp }}(\psi)\right]$

The best estimate of the actual rotation angle is assumed to be the $\psi$ value that maximizes the correlation coefficient $\rho$. The procedure to determine $\psi$ can cease according to a heuristic stopping rule (e.g., small $\rho$ improvements). Once determined the angle $\psi$, the correct axes rotation of the geographical coordinates is computed with Eq. (5), in order to fulfill the condition of having the corresponding axis parallel and the axes origin coincident w.r.t. the $C S_{r}$; thereafter, the radar data and results are interpolated on the DEM with Eq. (6).

In Fig. 6 we report the maps of OV and RV, highlighting different possible choices of the amplitude threshold for the correlation assessment.

\subsubsection{Data coupling}

The last step of the processing concerns the data coupling. The actual 3D motion vector $\mathbf{v}=\left[v_{x}, v_{y}, v_{z}\right]^{T}$ is represented into a local righthanded coordinate system with the following convention: $v_{x}, v_{y}$ are the horizontal components, respectively rightward and incoming increasing, while $v_{z}$ is the vertical downward-increasing component. With this convention, $v_{y}$ is mainly provided by the GBSAR measurements, while $v_{x}, v_{z}$ are mainly provided by the VBS. Therefore, their respective vectors can be written in their own CSs as

$$
\begin{gathered}
\mathbf{h}^{\mathbf{r}}=\left[0, h_{y}^{r}, 0\right]^{T} \\
\mathbf{h}^{\mathbf{v}}=\left[h_{x}^{v}, 0, h_{z}^{v}\right]^{T}
\end{gathered}
$$

Eq. (8) explicitly expresses that they measure only partial components of the actual 3D motion vector. As previously mentioned, if the 
radar and photographic data lie within a space where the corresponding axis are parallel, the three components are mutual perpendicular, thus they represent directly the three components of $\mathbf{v}$. On the other hand, when this condition is not satisfied, it is necessary to carry out a geometrical transformation in order to rotate one CS and make the correspondent axes parallel; the choice of the CS to be rotated is arbitrary.

It must be considered that in general, when a vector is rotated, all the three components assume non-null values; but for reconstructing the 3D motion only the linearly independent (i.e., orthogonal) components must be summed.

Therefore, applying the rotation matrix $\boldsymbol{R}$ to a generic motion vector h, we have

$\boldsymbol{R} \mathbf{h}=\mathbf{h}^{\prime}=\left[h_{x}^{\prime}, h_{y}^{\prime}, h_{z}^{\prime}\right]^{T}$

It is worth noting that the components measured by each device correspond to the actual components of $\mathbf{v}$ in their own CS; i.e., in the $C S_{v}: v_{x}=h_{x}$ and $v_{z}=h_{z}$, while in the $C S_{r}: v_{y}=h_{y}$. Therefore, if it rotated $C S_{r}$, the component $v_{y}=h_{y}^{r^{\prime}}$, while the other two components of $\mathbf{h}^{\mathbf{r}}$ are not considered to reconstruct $\mathbf{v}$ because their contribution is already present in $h_{x}^{v}$ and $h_{z}^{v}$. Thus, in $C S_{v}$ the motion vector is $\mathbf{v}=\left[h_{x}^{v}, h_{y}^{r^{\prime}}, h_{z}^{v}\right]^{T}$. Following the same principle, if the $C S_{v}$ was rotated, in the $C S_{r}$ we would have $\mathbf{v}=\left[h_{x}^{v \prime}, h_{y}^{r}, h_{z}^{v '}\right]^{T}$. Finally, once that the three motion components are obtained in a generic CS, $\mathbf{v}$ can be reported in $C S_{\text {geo }}$ adopting Eq. (9). Fig. 7 shows a scheme of the rotation in the specific case where the radar vector is transformed in $C S_{v}$.

In principle, a rotation in three dimensions is fulfilled around a reference axis, whose versor does not change during the transformation. In practice, the reference axis is usually unknown and it is necessary to operate a sequence of elemental rotations around the three canonical axes, conventionally corresponding to the geographical axes (Cai et al., 2011).

This solution has the advantage that, being the data georeferenced, the angles $(\psi, \theta, \phi)$ are known for every points within the interested areas, where $\psi$ is the angle included between the LOS and the geographical north, $\theta$ is the LOS elevation w.r.t. the horizontal plane and $\phi$ is the rotation of the $\mathrm{x}$-axis w.r.t. the horizontal plane, which is usually assumed zero. Therefore, Eq. (9) is applied to the motion vectors of each single georeferenced point, according with the corresponding known angles, therefore, $\boldsymbol{R}$ is a combination of elemental rotations in three dimensions.

It is worth noting that the application of a sequence of rotations

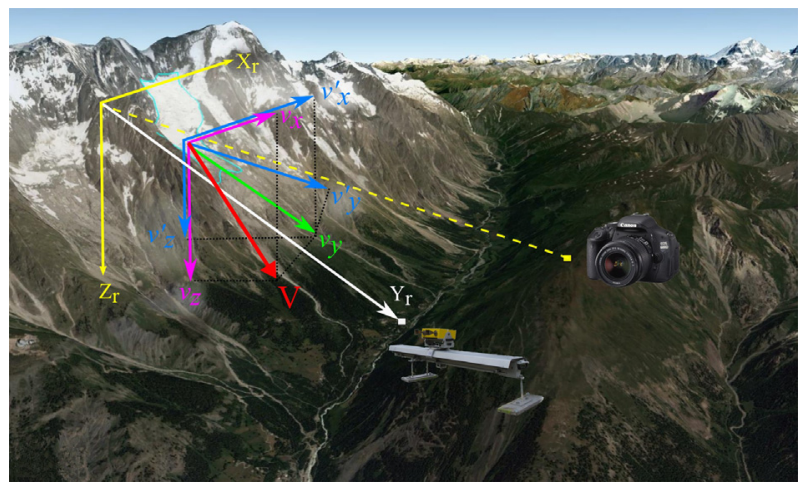

Fig. 7. Scheme of the vector transformation in the specific case in which the velocity vector measured by the radar is rotated in the coordinate system of the VBS. The components $v_{x}, v_{z}$ measured by the VBS are represented in magenta and the respective axes $X_{v}, Z_{v}$ orthogonal to the LOS (yellow dashed line) of the VBS are in yellow. The green arrow depicts the component $v_{y}$ measured by the GBSAR; the $Y_{r}$ axis of the $C S_{r}$ is colored in white and it coincides with the LOS of the GBSAR. The blue arrows indicate the components $v_{x}^{\prime}, v_{y}^{\prime}, v_{z}^{\prime}$ formed by the decomposition of $v_{x}$ when it is rotated in $C S_{v}$. The actual velocity vector $V=\left[v_{x}, v_{y}^{\prime}, v_{z}\right]$ is drawn in red. The white and yellow squares are the locations of the GBSAR and VBS respectively.

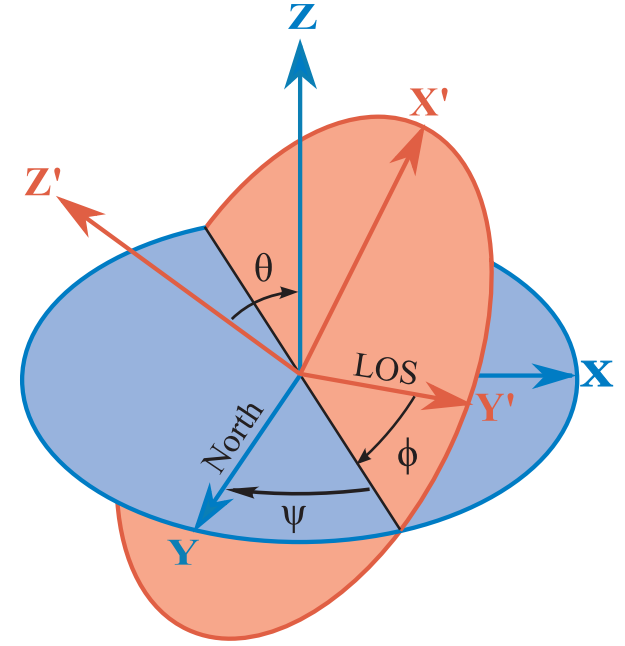

Fig. 8. Euler angles yaw, pitch and roll $(\psi, \theta, \phi)$ between a generic coordinate system $C S^{\prime}\left(X^{\prime}, Y^{\prime}, Z^{\prime}\right)$ and the canonical axes corresponding to the geographic coordinate system $C S_{\text {geo }}(X, Y, Z)$. The red and blue planes are the horizontal planes $\left(X^{\prime} Y^{\prime}, X Y\right)$ of the $C^{\prime} S^{\prime}$ and $C S_{\text {geo }}$ respectively. The sequence of the rotation angles to make coincide the $C S^{\prime}$ with $C S_{\text {geo }}$ is: $(i) \boldsymbol{R}_{y}(\phi),(i i) \boldsymbol{R}_{x}(\theta),(i i i) \boldsymbol{R}_{z}(\psi)$. The inverse rotation order transform the $C S_{\text {geo }}$ in $C S^{\prime}$.

around different axes is not commutative in three dimensions. To evaluate the unique sequence of rotations, we can consider $(\psi, \theta, \phi)$ as the Euler angles yaw, pitch and roll. With the present axes convention, the angle sequence for rotating the local $\mathrm{CS}$ in the $C S_{\text {geo }}$ is known, i.e., (i) rotation around the y-axis of the roll angle $\phi$, (ii) rotation around the $\mathrm{x}$-axis of the pitch angle $\theta$, and (iii) rotation around the z-axis of the yaw angle $\psi$ (Cai et al., 2011) (Fig. 8).

Therefore, the transformation introduced in Eq. (9) is explicitly expressed as the composite rotation given by the matrix multiply of six elemental rotations expressed by the following formula

$\boldsymbol{R} \mathbf{h}=\boldsymbol{R}_{y}\left(\psi_{r}\right) \boldsymbol{R}_{x}\left(\theta_{r}\right) \boldsymbol{R}_{z}\left(\phi_{r}\right) \boldsymbol{R}_{z}\left(\phi_{v}\right) \boldsymbol{R}_{x}\left(\theta_{v}\right) \boldsymbol{R}_{y}\left(\psi_{v}\right) \mathbf{h}=\mathbf{h}^{\prime}$

where $\boldsymbol{R}_{*}$ indicates the rotation axis, and $(\psi, \theta, \phi)$ are the Euler angles of the two CSs. That is, $\mathbf{h}$ is initially rotated from the first local CS in $C S_{g e o}$ and subsequently is transformed from $C S_{g e o}$ to the second local CS applying the inverse sequence of Euler rotations. We remind that the sign of the rotation angles for a right-handed coordinate system is positive for clockwise rotations and negative for counter-clockwise rotations.

\section{Sources of error}

The uncertainties that affected the results of our work mainly originated from two sources: (i) data processing and (ii) georeferencing.

To the former group belong the errors of the motion measurement carried out by the two single devices (i.e., VBS or GBSAR). We evaluated the measurement uncertainties by investigating the cumulative motion observed in selected areas corresponding to stable surfaces, i.e., bedrock (Dematteis et al., 2017). Since we expected a null cumulative motion, we associated the average cumulative motion to the measurement accuracy, while its variability, estimated as the mean absolute deviation (MAD), was associated to the precision. The results of the error analysis are presented in Table 2. Because the results of the ICC methods are expressed in pixel units, we converted the values in metric displacement, adopting an approximate GSD of $5.5 \mathrm{~cm}$ and $13.6 \mathrm{~cm}$ for the TELE and WIDE modules respectively (i.e., the GSD corresponding to the glacier average distance).

From the error analysis emerged that the performances of the ICC were comparable with those obtained in previous studies (Ahn and Box, 2010; Schwalbe and Maas, 2017; Travelletti et al., 2012). The slightly 
Table 2

Estimates of measurement accuracy and precision. The accuracy is computed as the mean cumulative motion on stable areas, while the precision is the mean absolute deviation. The values are computed on the 24 days-cumulative motion. $\mathrm{H}, \mathrm{V}$ stand for horizontal and vertical directions respectively, for the TELE and WIDE images.

\begin{tabular}{lll}
\hline & Accuracy $(\mathrm{cm})$ & Precision $(\mathrm{cm})$ \\
\hline TELE H & -0.23 & 0.78 \\
TELE V & 0.45 & 0.89 \\
WIDE H & -0.72 & 1.62 \\
WIDE V & -0.02 & 0.58 \\
GBSAR & -1.37 & 4.56 \\
\hline
\end{tabular}

worse precision of the GBSAR measurements can be ascribed to unresolved unwrapping errors. However, the relative precision is of the order of less than $1 \%$ of the maximum cumulative motion.

The second major error source belongs to the georeferencing uncertainty. For what concerns the image orthorectification, we carried out a 1000-iterations Monte Carlo simulation, randomly dividing the selected GCPs in two sets composed by one-half of the GCPs. With the former one, we calibrated the image orthorectification, while with the other group we estimated the mean absolute error (MAE) between the original location of the GCPs and the projected position that minimizes the squared error during the camera parameter bundle adjustment (Travelletti et al., 2012). We obtained mean MAEs of $21 \mathrm{px}$ and $12.5 \mathrm{px}$ for the TELE and WIDE images respectively. Using the whole set of GCPs we had MAEs of $18 \mathrm{px}$ and $12.4 \mathrm{px}$. Converting these values in metric units, we obtained a georeferencing error of approximately $0.99 \mathrm{~m}$ and $1.69 \mathrm{~m}$ (TELE and WIDE images respectively); similar uncertainties were obtained by (Liu et al., 2007). Finer results can be achieved by measuring the GCP coordinates with GPSs systems (Messerli and Grinsted, 2015) or performing multi-views photographic acquisition (e.g., stereoscopy, SFM) (Piermattei et al., 2016).

The radar data georeferencing was attained with the procedure described in Section 3.3.2. The method is based on the considerations that the RV and OV coincide and that the RV corresponds to the areas with high amplitude scattered signal. Both assumptions are strongly plausible, but the correspondence between RV and signal amplitude might suffer the arbitrary choice of the empirical amplitude threshold defined to detect the visible areas. In Fig. 9, we show the correlation coefficients computed between the $\mathrm{OV}$ and $\mathrm{RV}$ w.r.t. different rotation angles and thresholds (Fig. 6d depicts the areas on the DEM corresponding of different amplitude thresholds); it emerged that the proposed method is quite insensitive to the choice of the amplitude threshold. In fact, all the $\psi$ estimates that maximized the correlation coefficient $\rho$ obtained with different amplitude thresholds, lay within a range of $\pm 0.1^{\circ}$. Therefore, we judged the best estimate, $\widehat{\psi}$, the angle that provided the absolute maximum value of $\rho$ (i.e., $\widehat{\psi}=24.35^{\circ} \pm 0.1^{\circ}$ ). A rotation angle change of $\pm 0.1^{\circ}$ is equivalent to an azimuth uncertainty of $\pm 4.36 \mathrm{~m}$ at a distance of $2500 \mathrm{~m}$, while the range uncertainty is negligible.

We compared the precision and accuracy of the proposed method with the rotation angle assessment through the measurement of the radar edges coordinates. With the aid of GPSs, we carried out the measurements of the positions of two couples of points on the GBSAR rail, and consequently we derived the orientation of the instrument. The obtained estimates of $\psi$ are reported in Table 3 together with the corresponding uncertainty being the error ranging between 0.5 and $1 \mathrm{~cm}$ of each horizontal coordinate of the GPSs measurements.

From that comparison, it emerged that the value of the rotation angle evaluated with the correlation method is approximately the same resulting from the GPSs method, but in the latter case the uncertainty was approximately seven times greater. The geometric schemes of the two methods are different, and the measurement of the edge positioning with GPSs can amplify the error of the measurement. However,

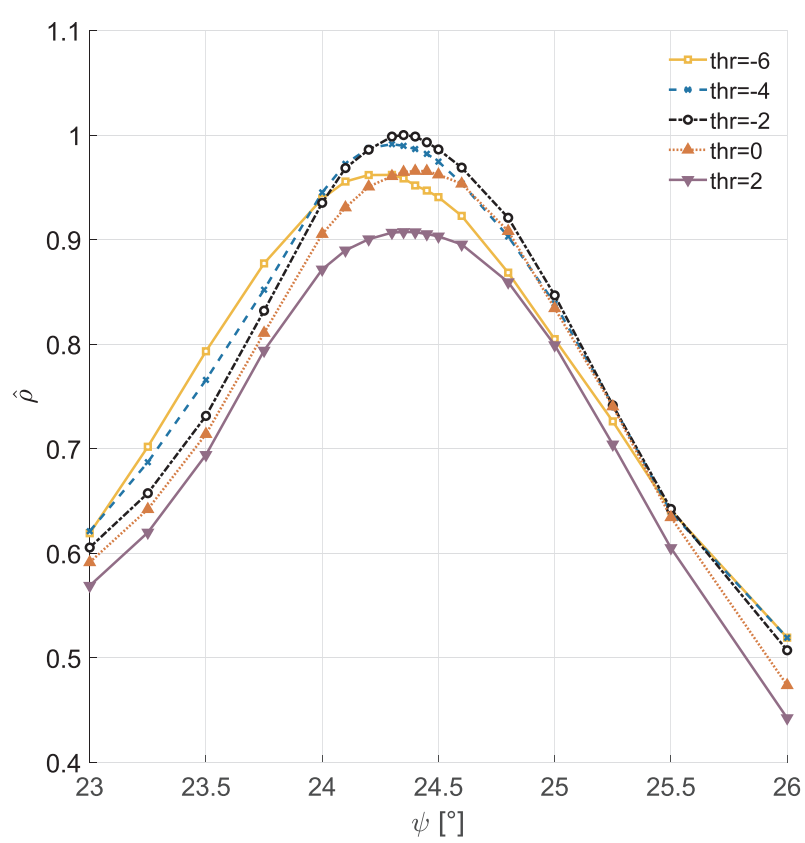

Fig. 9. Curves of normalized correlation coefficient between the visual and radar viewsheds vs. the rotation angle of the visual viewshed. The radar viewsheds were estimated adopting different thresholds of the mean amplitude signal in $\mathrm{dB}$.

Table 3

Rotation angles and corresponding uncertainties. GPS1 and GPS2 stand for the two measurements performed with the GPSs to estimate the rotation angle, GPSmean is the average of the two values. AngCorr indicates the angle assessment obtained with the correlation between radar and visual viewsheds.

\begin{tabular}{lll}
\hline & $\theta$ & Metric uncertainty \\
\hline GPS1 & $24.70^{\circ} \pm 0.3^{\circ}$ & $13.35 \mathrm{~m}$ (azimuth) @2500 m \\
GPS2 & $24.22^{\circ} \pm 0.7^{\circ}$ & $29.93 \mathrm{~m}$ (azimuth) @2500 m \\
GPSmean & $24.46^{\circ} \pm 0.75^{\circ}$ & $32.77 \mathrm{~m}$ (azimuth) @2500 m \\
AngCorr & $24.35^{\circ} \pm 0.1^{\circ}$ & $4.36 \mathrm{~m}$ (azimuth) @2500 m \\
\hline
\end{tabular}

Table 4

Map resolutions and uncertainties at the average distances sensor-to-glacier; i.e. $2500 \mathrm{~m}$ the GBSAR and $3800 \mathrm{~m}$ the VBS. The photographic images have the same resolution and uncertainty values in azimuth and range. The GBSAR range error is negligible.

\begin{tabular}{lllll}
\hline & $\begin{array}{l}\text { Azimuth } \\
\text { resolution (m) }\end{array}$ & $\begin{array}{l}\text { Azimuth } \\
\text { uncertainty (m) }\end{array}$ & $\begin{array}{l}\text { Range } \\
\text { resolution (m) }\end{array}$ & $\begin{array}{l}\text { Range } \\
\text { uncertainty (m) }\end{array}$ \\
\hline VBS tele & 3.51 & 0.99 & 3.51 & 0.99 \\
VBS wide & 8.65 & 1.68 & 8.65 & 1.68 \\
GBSAR & 11 & 4.36 & 0.43 & - \\
\hline
\end{tabular}

presently, to our knowledge, this is the common method used to estimate the orientation angle from remote. We proposed an alternative approach that can reduce the uncertainties of the georeferencing.

In Table 4 we summarize the values of the map resolutions of the different systems and the corresponding georeferencing errors. All the values were of comparable size of the order of a few metres; therefore, we considered the georeferencing uncertainties adequate for the data coupling.

\section{Results}

In this section, we report the results obtained by the single remote sensing devices, i.e., the VBS and the GBSAR; these data represent partial components of the surface motion. The actual 3-dimensional 


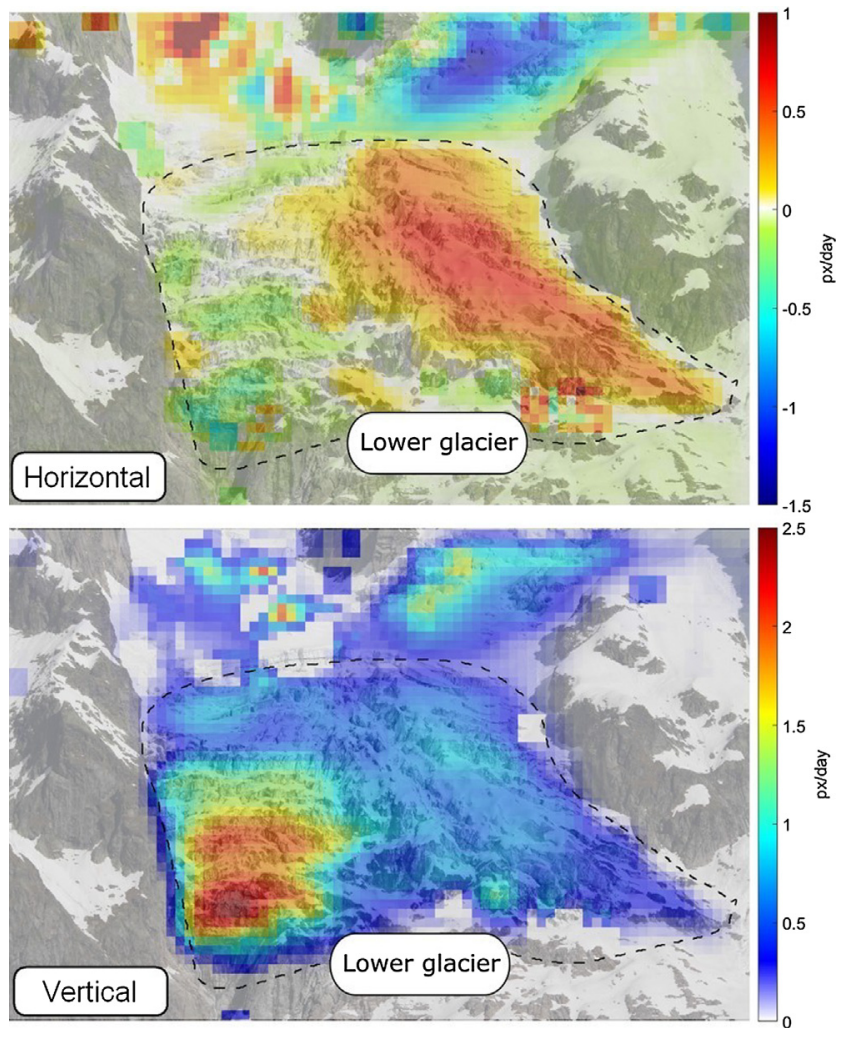

Fig. 10. Upper image: map of the horizontal component of the velocity obtained by ICC of the WIDE images; positive values represent rightward motion, while negative represent leftward movement. Lower image: map of the vertical component of the velocity obtained by ICC of the WIDE images. A dashed line surrounds the lower portion of the glacier (i.e., the investigated part). kinematics is achieved by the coupling of the two single results, and it is shown below.

In Fig. 10, we present the horizontal and vertical components of the velocity of the WIDE images, as they are obtained by the ICC processing; the raw results of the ICC are expressed in $\mathrm{px} /$ day because the metric conversion is possible only after the image orthorectification.

Fig. 11 depicts the mean daily motion measured by the GBSAR (in $\mathrm{rad} /$ day). It is evident the difference of the data representations between the two processing methods.

The actual 3-dimensional surface kinematics was produced by the coupling of the visual and interferometric data. Fig. 12 shows the daily velocity map projected on the orthophoto. The colours of motion field represent the module of the velocity, while the arrows indicate the direction and versus; the arrow length is proportional to the module.

The 3D results can be achieved only in the areas visible by both the devices. Therefore, the blank areas correspond to the points in shade from the perspective of the devices; in particular, due to its location the GBSAR had a limited view of the eastern tongue. Note that the 3D motion is mostly influenced by the $v_{z}$ and $v_{y}$ components that show a similar pattern; $v_{x}$ is almost irrelevant for it has quite low values.

The velocity field was estimated with a daily frequency allowing the precise observation of the evolution of the actual surface kinematics. That offered the opportunity to identify the occurrence of short-term phenomena; i.e., we registered a deceleration in the last period of the survey.

The knowledge of the three velocity components allowed analyzing in detail the behaviour (e.g., the motion direction) of particular areas of interest. Interestingly, we observed that the motion vector was not uniformly parallel to the local glacier surface; on the contrary, in the steepest areas the $v_{y}$ component was dominant, while $v_{z}$ was more relevant in the gentler parts. Usually, if a recent DEM is available, the 3D motion is estimated by adjusting the 1D or 2D measurements according to the local slope (Gabrieli et al., 2016; Giordan et al., 2016; Huang and Li, 2011; Luzi et al., 2007). Though, as we proved, this assumption might be misleading in particular cases.

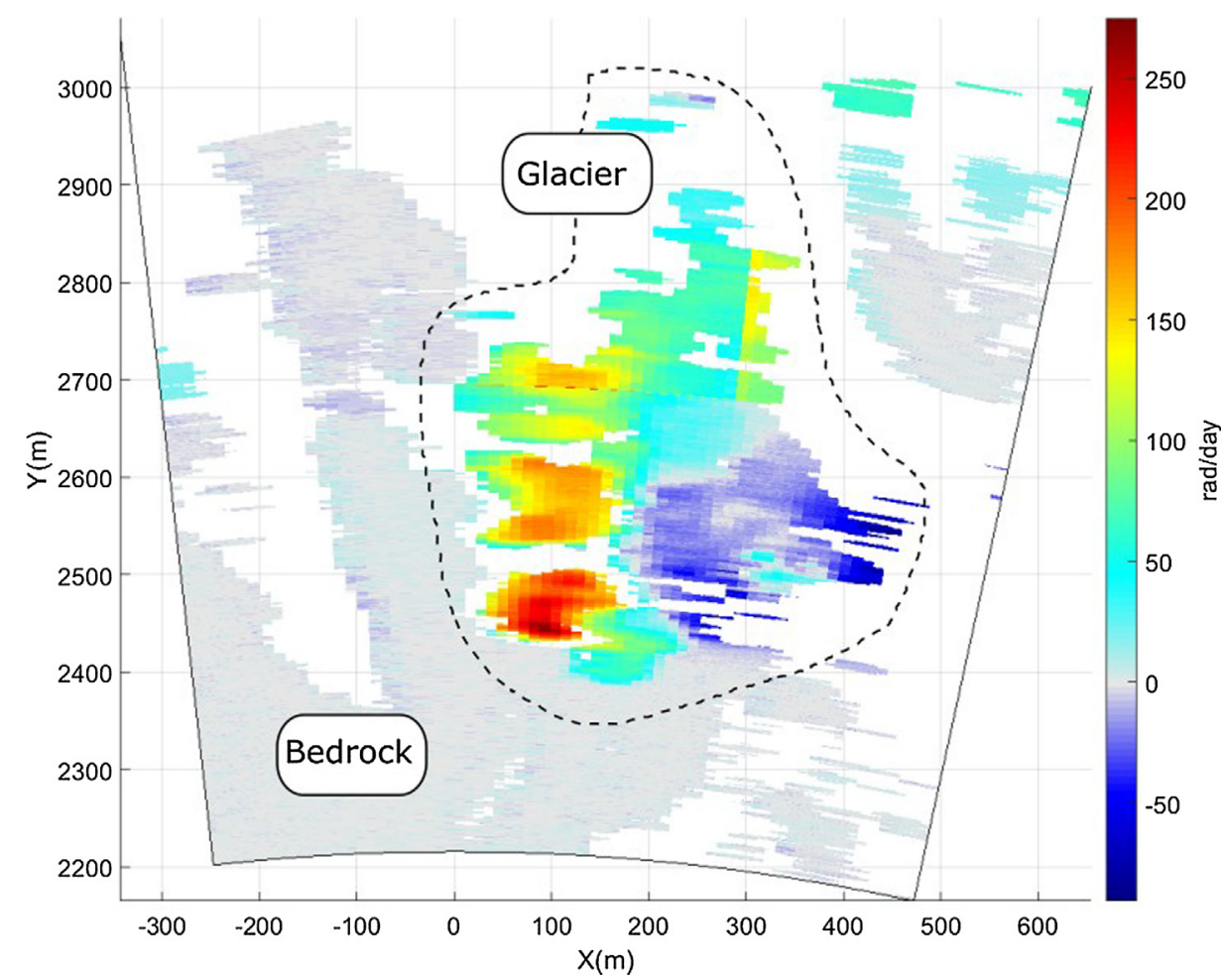

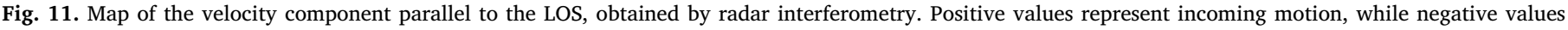
represent motion going away. A dashed line surrounds the glacier; the grey areas indicate the bedrock. 


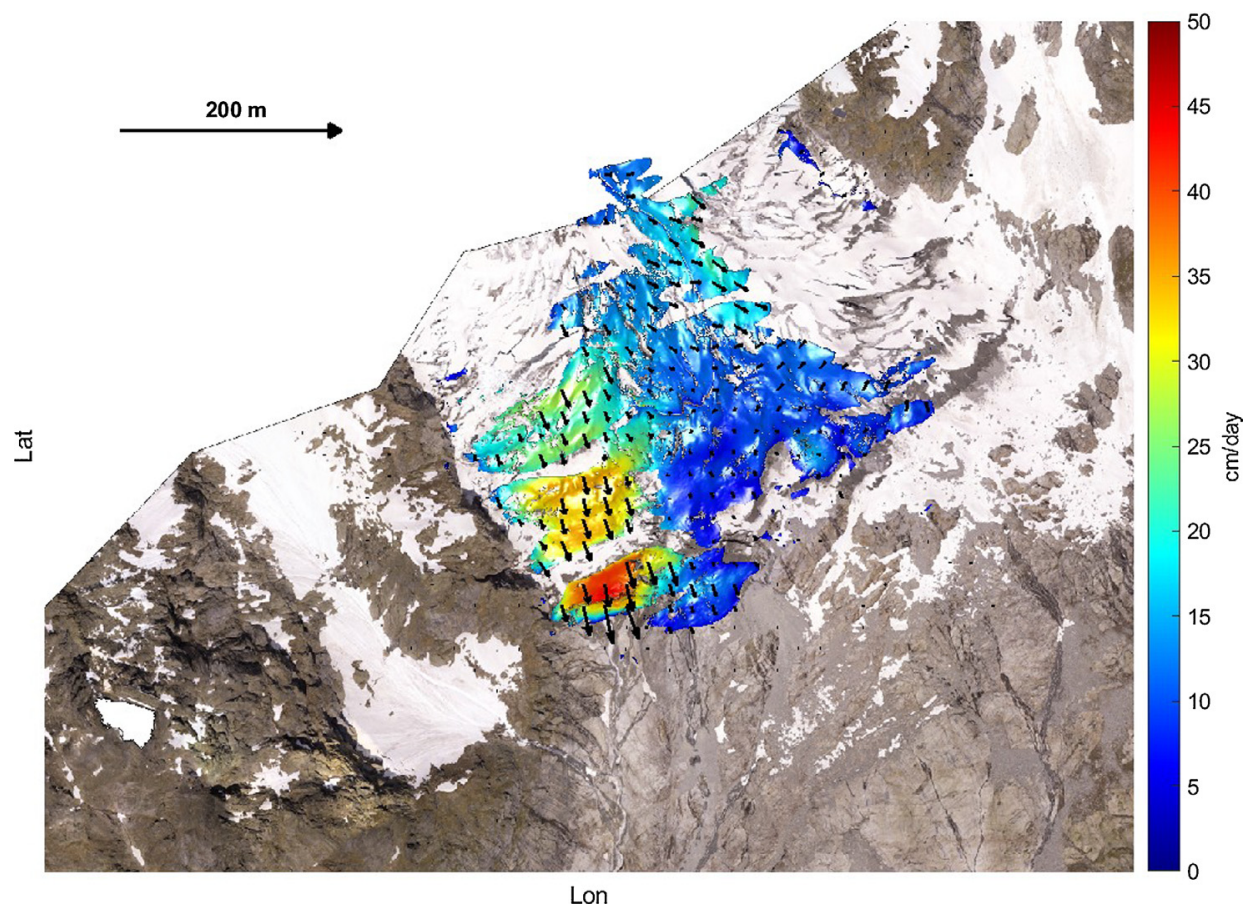

Fig. 12. Velocity field of the surface kinematics. Colours and arrows represent the velocity module and direction respectively.

In the present situation, the surface displacements were not parallel to the local slope probably because the vertical areas corresponded to single ice bodies (e.g., seracs and ice block faces) that slid according with the entire glacier body along the global valley slope. Whereas, in the flatter regions, the surface deformations (i.e., ice creeping) are a relevant component of the motion, thus the vertical component is dominant. The same phenomena might be valid also in situations where the investigated surface is characterized by the presence of prominent volumes moving as single bodies (e.g., icefalls, deep gravitational deformations or toppling-affected areas).

\section{Conclusions}

In this paper, we presented an innovative methodology that couples the partial measurements of the surface kinematics obtained through terrestrial radar interferometry (TRI) and image cross-correlation (ICC) as they can detect complementary motion components.

The technique we developed allows at estimating the spatio-temporal evolution (daily frequency) of the actual 3-dimensional surface kinematics, providing module, direction and versus of the motion field. It is possible to employ it purely from remote from medium range (i.e., a few thousand metres). Therefore, that approach is particularly suited in situations where the access to the investigated area is difficult or hazardous. Moreover, the method can be applied either when the two remote sensing instruments are placed in coincident or different locations. That feature lends our approach a flexible logistic.

The major issue concerns the data georeferencing, which is accomplished with the support of a DEM. The visual images are orthorectified through bundle adjustment using a set of ground control points (GCPs) manually selected on the scene. The orthorectification process also provides the metric scaling of the data. A relevant characteristic of the general method is that the data merging is quite insensitive to possible morphological changes of the surface, mainly because the pixel-to-metric conversion depends only on the sensor-to-target distance, whose variation is negligible.

We propose a new approach to georeference the radar data purely from remote. The idea consists in estimating the unknown transformation parameter (i.e., the rotation angle) that maximize the spatial correlation between the optical and the radar viewsheds; the radar viewshed is evaluated form the analysis of the amplitude of the backscattered signal. Once the data are georeferenced, the orthogonal components of the motion are summed.

We applied the technique to the dataset collected during a survey campaign of the Planpincieux glacier, in the Italian side of the Mont Blanc massif, carried out on 4-27 September 2015.

We acquired the data with a ground-based synthetic aperture radar (GBSAR) and a monoscopic visual-based system (VBS). The measurements of the cumulative motion with both the instruments revealed millimeter accuracy and precision. The georeferencing uncertainty was estimated in a few metres; that is comparable with the resolution of the maps of the results. Therefore, we judged the georeferencing method satisfying for the data coupling.

Besides the georeferencing error, the main limitations of the method are those related to the single remote sensing system; e.g., the ICC method requires the manual selection of the images to be processed, it works only during suited visibility conditions, and it suffers the shadow-light effects. The terrestrial radar interferometry drawback is primarily connected to the high financial costs and the relatively limited portability of the instrumentation.

\section{References}

Aguilar, M.A., Agüera, F., Aguilar, F.J., Carvajal, F., 2008. Geometric accuracy assessment of the orthorectification process from very high resolution satellite imagery for Common Agricultural Policy purposes. Int. J. Remote Sens. 29, 7181-7197.

Ahn, Y., Box, J.E., 2010. Glacier velocities from time-lapse photos: technique development and first results from the Extreme Ice Survey (EIS) in Greenland. J. Glaciol. 56, 723-734. http://dx.doi.org/10.3189/002214310793146313.

Allstadt, K.E., Shean, D.E., Campbell, A., Fahnestock, M., Malone, S.D., 2015. Observations of seasonal and diurnal glacier velocities at Mount Rainier, Washington, using terrestrial radar interferometry. The Cryosphere 9, 2219-2235.

Arenson, L.U., Kääb, A., O'Sullivan, A., 2016. Detection and analysis of ground deformation in permafrost environments. Permafr. Periglac. Process. 27, 339-351.

Bamler, R., Hartl, P., 1998. Synthetic aperture radar interferometry. Inverse Probl. 14, R1.

Bitelli, G., Dubbini, M., Zanutta, A., 2004. Terrestrial laser scanning and digital photogrammetry techniques to monitor landslide bodies. Int. Arch. Photogramm. Remote Sens. Spat. Inf. Sci. 35, 246-251.

Caduff, R., Schlunegger, F., Kos, A., Wiesmann, A., 2015. A review of terrestrial radar interferometry for measuring surface change in the geosciences. Earth Surf. Process. Landf. 40, 208-228. 
Cai, G., Chen, B.M., Lee, T.H., 2011. Unmanned Rotorcraft Systems. Springer Science \& Business Media.

Casu, F., Manconi, A., Pepe, A., Lanari, R., 2011. Deformation time-series generation in areas characterized by large displacement dynamics: the SAR amplitude pixel-offset SBAS technique. IEEE Trans. Geosci. Remote Sens. 49, 2752-2763.

Cheng, D., Prasad, D.K., Brown, M.S., 2014. Illuminant estimation for color constancy: why spatial-domain methods work and the role of the color distribution. JOSA A 31, 1049-1058.

Costantini, M., 1998. A novel phase unwrapping method based on network programming. IEEE Trans. Geosci. Remote Sens. 36, 813-821.

Debella-Gilo, M., Kääb, A., 2011. Sub-pixel precision image matching for measuring surface displacements on mass movements using normalized cross-correlation. Remote Sens. Environ. 115, 130-142.

Delacourt, C., Allemand, P., Berthier, E., Raucoules, D., Casson, B., Grandjean, P., Pambrun, C., Varel, E., 2007. Remote-sensing techniques for analysing landslide kinematics: a review. Bull. Société Géologique Fr. 178, 89-100. http://dx.doi.org/10. 2113/gssgfbull.178.2.89.

Dematteis, N., Luzi, G., Giordan, D., Zucca, F., Allasia, P., 2017. Monitoring Alpine glacier surface deformations with GB-SAR. Remote Sens. Lett. 8, 947-956.

Faillettaz, J., Funk, M., Vincent, C., 2015. Avalanching glacier instabilities: review on processes and early warning perspectives. Rev. Geophys. 53, 203-224.

Fallourd, R., Vernier, F., Friedt, J.-M., Martin, G., Trouvé, E., Moreau, L., Nicolas, J.-M., 2010. Monitoring temperate glacier with high resolution automated digital cameras-application to the Argentière Glacier. In: PCV 2010, ISPRS Commission III Symposium.

Ferretti, A., Prati, C., Rocca, F., 2001. Permanent scatterers in SAR interferometry. IEEE Trans. Geosci. Remote Sens. 39, 8-20.

Fukuzono, T., 1985. A method to predict the time of slope failure caused by rainfall using the inverse number of velocity of surface displacement. Landslides 22, 8-13_1.

Gabrieli, F., Corain, L., Vettore, L., 2016. A low-cost landslide displacement activity assessment from time-lapse photogrammetry and rainfall data: application to the Tessina landslide site. Geomorphology 269, 56-74.

Giordan, D., Allasia, P., Dematteis, N., Dell'Anese, F., Vagliasindi, M., Motta, E., 2016. A low-cost optical remote sensing application for glacier deformation monitoring in an alpine environment. Sensors 16, 1750.

Gui, L.C., Merzkirch, W., 1996. A method of tracking ensembles of particle images. Exp. Fluids 21, 465-468.

Guizar-Sicairos, M., Thurman, S.T., Fienup, J.R., 2008. Efficient subpixel image registration algorithms. Opt. Lett. 33, 156-158.

Hart, D.P., 1998. The elimination of correlation errors in PIV processing. In: 9th International Symposium on Applications of Laser Techniques to Fluid Mechanics, pp. 13-16.

Huang, L., Li, Z., 2011. Comparison of SAR and optical data in deriving glacier velocity with feature tracking. Int. J. Remote Sens. 32, 2681-2698.

Iglesias, R., Fabregas, X., Aguasca, A., Mallorqui, J.J., López-Martínez, C., Gili, J.A., Corominas, J., 2014. Atmospheric phase screen compensation in ground-based SAR with a multiple-regression model over mountainous regions. IEEE Trans. Geosci. Remote Sens. 52, 2436-2449.

Kääb, A., Gudmundsson, G.H., Hoelzle, M., 1998. Surface deformation of creeping mountain permafrost. Photogrammetric investigations on rock glacier Murtèl, Swiss Alps. In: Proceedings of the 7th International Conference on Permafrost, pp. 531-537.

Kääb, A., Huggel, C., Fischer, L., Guex, S., Paul, F., Roer, I., Salzmann, N., Schlaefli, S. Schmutz, K., Schneider, D., et al., 2005. Remote sensing of glacier-and permafrostrelated hazards in high mountains: an overview. Nat. Hazards Earth Syst. Sci. 5, 527-554.

Keane, R.D., Adrian, R.J., 1992. Theory of cross-correlation analysis of PIV images. Appl. Sci. Res. 49, 191-215.

Kenner, R., Bühler, Y., Delaloye, R., Ginzler, C., Phillips, M., 2014. Monitoring of high alpine mass movements combining laser scanning with digital airborne photogrammetry. Geomorphology 206, 492-504.

Leprince, S., Berthier, E., Ayoub, F., Delacourt, C., Avouac, J.-P., 2008. Monitoring earth surface dynamics with optical imagery. Eos Trans. Am. Geophys. Union 89, 1-2. http://dx.doi.org/10.1029/2008EO010001.

Lim, M., Petley, D.N., Rosser, N.J., Allison, R.J., Long, A.J., Pybus, D., 2005. Combined digital photogrammetry and time-of-flight laser scanning for monitoring cliff evolution. Photogramm. Rec. 20, 109-129.

Lingua, A., Piatti, D., Rinaudo, F., 2008. Remote monitoring of a landslide using an integration of GB-INSAR and LIDAR techniques. Int. Arch. Photogramm. Remote Sens. Spat. Inf. Sci. 37, 133-139.

Liu, X., Zhang, Z., Peterson, J., Chandra, S., 2007. LiDAR-derived high quality ground control information and DEM for image orthorectification. GeoInformatica 11, 37-53.

Luhmann, T., 2009. Precision potential of photogrammetric 6DOF pose estimation with a single camera. ISPRS J. Photogramm. Remote Sens. 64, 275-284.

Luzi, G., Pieraccini, M., Mecatti, D., Noferini, L., Guidi, G., Moia, F., Atzeni, C., 2004. Ground-based radar interferometry for landslides monitoring: atmospheric and instrumental decorrelation sources on experimental data. IEEE Trans. Geosci. Remote
Sens. 42, 2454-2466

Luzi, G., Pieraccini, M., Mecatti, D., Noferini, L., Macaluso, G., Tamburini, A., Atzeni, C. 2007. Monitoring of an alpine glacier by means of ground-based SAR interferometry. IEEE Geosci. Remote Sens. Lett. 4, 495-499.

Malet, J.P., Ferhat, G., Ulrich, P., Boetzlé, P., Travelletti, J., 2016. The French National Landslide Observatory OMIV-Monitoring surface displacement using permanent GNSS, photogrammetric cameras and terrestrial LiDAR for understanding the landslide mechanisms.

Manconi, A., Allasia, P., Giordan, D., Baldo, M., Lollino, G., Corazza, A., Albanese, V., 2013. Landslide 3D surface deformation model obtained via RTS measurements. In: Landslide Science and Practice. Springer, pp. 431-436.

Marambio, A., Pucci, B., Jungner, A., Núnez, M., Buill, F., 2009. Terrestrial laser scanner, terrestrial synthetic aperture radar and topographic data: an integration proposal. In: Proc 8th Int. Geomat. Week Barc.

Messerli, A., Grinsted, A., 2015. Image georectification and feature tracking toolbox: ImGRAFT. Geosci. Instrum. Methods Data Syst. 4, 23.

Monserrat, O., Crosetto, M., 2008. Deformation measurement using terrestrial laser scanning data and least squares 3D surface matching. ISPRS J. Photogramm. Remote Sens. 63, 142-154.

Monserrat, O., Crosetto, M., Luzi, G., 2014. A review of ground-based SAR interferometry for deformation measurement. ISPRS J. Photogramm. Remote Sens. 93, 40-48.

Noferini, L., Mecatti, D., Macaluso, G., Pieraccini, M., Atzeni, C., 2009. Monitoring of Belvedere Glacier using a wide angle GB-SAR interferometer. J. Appl. Geophys. 68, 289-293.

Noferini, L., Pieraccini, M., Mecatti, D., Luzi, G., Atzeni, C., Tamburini, A., Broccolato, M., 2005. Permanent scatterers analysis for atmospheric correction in ground-based SAR interferometry. IEEE Trans. Geosci. Remote Sens. 43, 1459-1471.

Piermattei, L., Carturan, L., de Blasi, F., Tarolli, P., Fontana, G.D., Vettore, A., Pfeifer, N., 2016. Suitability of ground-based SfM-MVS for monitoring glacial and periglacial processes. Earth Surf. Dyn. 4.

Pralong, A.J.W., 2005. On the instability of hanging glaciers: Study on the fracture processes and the predictability of failures (PhD Thesis). ETH, Zürich.

Pust, O., 2000. Piv: Direct cross-correlation compared with fft-based cross-correlation. In: Proceedings of the 10th International Symposium on Applications of Laser Techniques to Fluid Mechanics, Lisbon, Portugal. p. 114.

Raucoules, D., De Michele, M., Malet, J.-P., Ulrich, P., 2013. Time-variable 3D ground displacements from high-resolution synthetic aperture radar (SAR). Application to La Valette landslide (South French Alps). Remote Sens. Environ. 139, 198-204.

Riesen, P., Strozzi, T., Bauder, A., Wiesmann, A., Funk, M., 2011. Short-term surface ice motion variations measured with a ground-based portable real aperture radar interferometer. J. Glaciol. 57, 53-60.

Roncella, R., Forlani, G., Fornari, M., Diotri, F., 2014. Landslide monitoring by fixed-base terrestrial stereo-photogrammetry. ISPRS Ann. Photogramm. Remote Sens. Spat Inf. Sci. 2, 297.

Rosen, P.A., Hensley, S., Joughin, I.R., Li, F.K., Madsen, S.N., Rodriguez, E., Goldstein, R.M., 2000. Synthetic aperture radar interferometry. Proc. IEEE 88, 333-382.

Scaioni, M., Corti, M., Diolaiuti, G., Fugazza, D., Cernuschi, M., 2017. Local and general monitoring of Forni glacier (Italian Alps) using multi-platform structure-from-motion photogrammetry. Int. Arch. Photogramm. Remote Sens. Spat. Inf. Sci. 42.

Scherler, D., Leprince, S., Strecker, M.R., 2008. Glacier-surface velocities in alpine terrain from optical satellite imagery_accuracy improvement and quality assessment. Remote Sens. Environ. 112, 3806-3819.

Schwalbe, E., Maas, H.-G., 2017. The determination of high-resolution spatio-temporal glacier motion fields from time-lapse sequences. Earth Surf. Dyn. 5, 861.

Sibson, R., 1981. A brief description of natural neighbor interpolation. Interpret. Multivar. Data 21-36.

Strozzi, T., Luckman, A., Murray, T., Wegmuller, U., Werner, C.L., 2002. Glacier motion estimation using SAR offset-tracking procedures. IEEE Trans. Geosci. Remote Sens. 40, 2384-2391.

Tapete, D., Casagli, N., Luzi, G., Fanti, R., Gigli, G., Leva, D., 2013. Integrating radar and laser-based remote sensing techniques for monitoring structural deformation of archaeological monuments. J. Archaeol. Sci. 40, 176-189.

Thielicke, W., Stamhuis, E., 2014. PIVlab-towards user-friendly, affordable and accurate digital particle image velocimetry in MATLAB. J. Open Res, Softw, pp. 2.

Travelletti, J., Delacourt, C., Allemand, P., Malet, J.-P., Schmittbuhl, J., Toussaint, R., Bastard, M., 2012. Correlation of multi-temporal ground-based optical images for landslide monitoring: application, potential and limitations. ISPRS J. Photogramm. Remote Sens. 70, 39-55.

Westerweel, J., Scarano, F., 2005. Universal outlier detection for PIV data. Exp. Fluids 39, 1096-1100.

White, D.J., Take, W.A., Bolton, M.D., 2003. Soil deformation measurement using particle image velocimetry (PIV) and photogrammetry. Geotechnique 53, 619-631.

Willert, C.E., Gharib, M., 1991. Digital particle image velocimetry. Exp. Fluids 10, 181-193.

Wright, T.J., Parsons, B.E., Lu, Z., 2004. Toward mapping surface deformation in three dimensions using InSAR. Geophys. Res, Lett, pp. 31. 